\title{
Correction: Vaidyanathan et al. Are We Eating Our Way to Prostate Cancer-A Hypothesis Based on the Evolution, Bioaccumulation, and Interspecific Transfer of miR-150. Non-Coding RNA 2016, 2, 2.
}

\author{
Venkatesh Vaidyanathan ${ }^{1, *}$, Vetrivhel Krishnamoorthy ${ }^{1}$, Nishi Karunasinghe ${ }^{2}$, Anower Jabed ${ }^{3}$, \\ Radha Pallati ${ }^{1}$, Chi Hsiu-Juei Kao ${ }^{1}$, Alice Wang ${ }^{1}$, Gareth Marlow ${ }^{4}$ and Lynnette R. Ferguson ${ }^{1,2}$ \\ 1 Discipline of Nutrition and Dietetics, FM \& HS, University of Auckland, Auckland 1023, New Zealand; \\ vkri468@aucklanduni.ac.nz (V.K.); rpal628@aucklanduni.ac.nz (R.P.); b.kao@auckland.ac.nz (C.H.-J.K.); \\ alice.wang@auckland.ac.nz (A.W.); 1.ferguson@auckland.ac.nz (L.R.F.) \\ 2 Auckland Cancer Society Research Centre, Auckland 1023, New Zealand; n.karunasinghe@auckland.ac.nz \\ 3 Department of Molecular Medicine and Pathology, FM \& HS, University of Auckland, Auckland 1023, \\ New Zealand; a.jabed@auckland.ac.nz \\ 4 Experimental Cancer Medicine Centre, Cardiff University, Cardiff, CF14 4XN, UK; MarlowG@cardiff.ac.uk \\ * Correspondence: v.vaidyanathan@auckland.ac.nz; Tel.: +64-9-923-6513; Fax: +64-9-373-7502
}

Academic Editor: George A. Calin

Published: 20 June 2016

The authors wish to make the following correction to this paper [1]. Reference 28 has been renumbered to 29, and the following affirmation in the original text: "Dezhong et al. have demonstrated that miR-150 was overexpressed in PCa cells at both the mRNA and protein levels" has been substituted by: "Waltering et al., 2011, reported that miR-150* along with three other miRNAs, namely, miR-10a, miR-141, and miR-1225-5p, among 17 miRNAs, expressed to have $>1.5$ - fold changes at the expression levels in PCa cells and in a minimum of two intact-castration xenograft pair. It has been shown that miR-150 was overexpressed in PCa cells."

Thereby, we include the research done by another group-Waltering et al., 2011—who also found interesting results working on PCa cell-lines and xenografts and the effect of miRNAs (reference no. 31 in the edited version).

References 29 and 30 were initially cited unintentionally on page 2 of the article. These references have been deleted from page number 2 and renumbered to 38 and 39 on page 3.

\section{Reference}

1. Vaidyanathan, V.; Krishnamoorthy, V.; Karunasinghe, N.; Jabed, A.; Pallati, R.; Kao, C.H.; Wang, A.; Marlow, G.; Ferguson, L.R. Are We Eating Our Way to Prostate Cancer-A Hypothesis Based on the Evolution, Bioaccumulation, and Interspecific Transfer of miR-150. ncRNA 2016, 2, 2. [CrossRef]

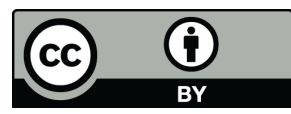

(C) 2016 by the authors; licensee MDPI, Basel, Switzerland. This article is an open access article distributed under the terms and conditions of the Creative Commons Attribution (CC-BY) license (http:/ / creativecommons.org/licenses/by/4.0/). 